\title{
Numerical Representation of Bodner Viscoplastic Constitutive Model
}

\author{
By Faysal A. Kolkailah and Andrew J. McPhate
}

\begin{abstract}
Nonlinear analyses of structural components are normally carried out by finite element codes making use of constitutive theories in which the material response is separated into the two important groups of phenomena known as ratedependent "creep" and rate-independent "plasticity." A number of viscoplastic constitutive theories in which creep and plasticity effects are combined into a unified plastic strain model have recently been proposed and are still undergoing active development. In this paper, the constitutive equations of the Bodner-Partom model are used to present the time dependent, inelastic properties of Inconel 718 at $650^{\circ} \mathrm{C}$. This representation covers a wide range of loading conditions. The developed numerical technique to establish the Bodner parameters is based on simulation with fourth-order Runge-Kutta integration coupled to a least square measure for good curve fitting to a series of tests. To determine the eight Bodner parameters, an error function consisting of the square of the difference between experimental and model strains was minimized in the time domain by a direct search method. The specific material parameters for the Bodner model were determined to best fit sets of tensile and creep data. The parameters so pbtained are in good agreement with those obtained by other investigators. The present parameters generate better response curves than those from graphical methods used in earlier investigations.
\end{abstract}

\section{INTRODUCTION}

Inconel 718 is a high temperature superalloy specially developed for lowcycle-fatigue limited components operating at high temperature and severe stress and in a hostile environment. It is important to determine and properly characterize its time dependent inelastic properties. A number of viscoplastic constitutive theories in which creep and plasticity effects are combined into a unified plastic strain model have recently been proposed and are still undergoing active development. One of these theories, employed in this study, is the constitutive theory of Bodner and Partom (1975).

Bodner used the constitutive equations of Bodner and Partom to represent the inelastic behavior of Rene 95 at $650^{\circ} \mathrm{C}\left(1,200^{\circ} \mathrm{F}\right)$. Stouffer (1981) used the state variable constitutive equations of Bodner and Partom to calculate the mechanical response of IN 100 at $730^{\circ} \mathrm{C}\left(1,350^{\circ} \mathrm{F}\right)$. Hinnerichs et al. (1982) estimated the material constants in IN 100 by using Bodner and Partom constitutive equations and then analyzed the creep crack growth in a nickel alloy at $730^{\circ} \mathrm{C}\left(1,350^{\circ} \mathrm{F}\right)$. Milly and Allen (1982) represented the experimental data for Inconel 718 at $650^{\circ} \mathrm{C}\left(1,200^{\circ} \mathrm{F}\right)$, from which the material constants were determined by the method given by Stouffer and Bodner (1981). The Bodner constitutive equations were then applied in a time do- 
main simulation. Milly compared the theory and experimental data and concluded the overall behavior was good.

\section{Constitutive Theory of Bodner and Partom}

The constitutive theory of Bodner and Partom (1975) is based on an assumption of small strain and that the total strain rate $\dot{\epsilon}^{\prime}(t)$ is separable into elastic (reversible), $\dot{\epsilon}^{e}(t)$, and plastic (irreversible), $\dot{\epsilon}^{p}(t)$, components, both nonzero for all loading/unloading conditions:

$\dot{\epsilon}^{\prime}(t)=\dot{\boldsymbol{\epsilon}}^{e}(t)+\dot{\boldsymbol{\epsilon}}^{p}(t)$.

with

$\dot{\epsilon}^{e}(t)=\frac{\dot{\sigma}(t)}{E}$

For the plastic strain rate, $\dot{\epsilon}^{p}(t)$, the specific representation used by Bodner and Partom was given by

$\dot{\epsilon}^{p}(t)=\frac{2}{\sqrt{3}} \frac{\sigma(t)}{|\sigma(t)|} D_{0} \exp \left[\frac{1}{2}\left(\frac{Z(t)}{(t)}\right)^{2 n}\left(\frac{n+1}{n}\right)\right] \ldots$

where $\sigma(t)=$ current value of the stress; $D_{0}=$ constant representing the limiting value of the plastic strain rate in shear that is generally taken at $10^{4}$ $\mathrm{s}^{-1}$, except for conditions of very high rates of strain; $n=$ constant related to the viscosity of the dislocation motion that controls the strain rate sensitivity; $Z(t)=$ hardness, state variable measure of overall resistance to plastic flow.

The evolution equation, i.e., history dependence, of the plastic state variable is generally sought in the form of a differential equation for hardening rate, $\dot{Z}$, that depends on stress, temperature, and hardness. A more specific representation is based on the concept that only the plastic work rate, $\dot{W}^{p}$, and current hardness, $Z$, control $\dot{Z}$. The complete expression for $Z$ can be written (Bodner and Partom 1975)

$\left(Z-Z_{1}\right)=\left(Z_{1}-Z_{0}\right) \exp \left(-m W^{p}\right)$

where $m=$ material constant controlling the rate of work hardening; $Z_{1}=$ saturation value of $Z$ for large $W^{p}$, i.e., it is the maximum value of $Z$, which is taken to be a material constant; $Z_{0}=$ initial value of $Z$, corresponding to the reference state from which $W^{p}$ is measured $\left(0 \leqq Z_{0} \leqq Z_{1}\right)$.

$\dot{W}^{p}=\sigma \dot{\epsilon}^{p}+\frac{\dot{Z}_{\text {recovery }}}{m\left(Z_{1}-Z\right)}$.

where,

$\dot{Z}_{\text {recovery }}=-A Z_{1}\left(\frac{Z-Z_{i}}{Z_{1}}\right)^{r}$

Therefore, the complete expression for $\dot{Z}$ can be written as

$\dot{Z}=m\left(Z_{1}-Z\right) \sigma \dot{\epsilon}^{p}-A Z_{1}\left(\frac{Z-Z_{i}}{Z_{1}}\right)^{r}$ 
where $A=$ coefficient controlling the rate of hardening recovery; $r=$ exponent controlling the rate of hardening recovery; $Z_{i}=$ the state variable value corresponding to the complete nonwork hardened condition, a function of temperature. In Eq. 7, the second term is hardening recovery, negligible during rapid load histories. Therefore, for long time response, such as creep, the second term in Eq. 7 is necessary, but during a tensile test, which is fast compared to creep, Eq. 7 reduces to the first term only. For a tensile test, Eq. 7 becomes

$\dot{Z}=m\left(Z_{1}-Z\right) \dot{W}^{p}$

In this case, $\dot{W}^{p}$ is determined by only

$\dot{W}^{p}=\sigma \dot{\epsilon}^{p}$.

Since the test data can be resolved into $\sigma$ and $\dot{\epsilon}^{p}$, Eq. 3 is solved for $Z$, a function of $\sigma$ and $\dot{\epsilon}^{p}$, as follows:

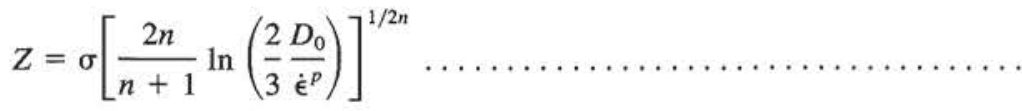

To determine the viscoplastic material constants in these constitutive equations, the constants are considered to be in two groups, creep response and short time response. The short time response constants are $D_{0}, n, m, Z_{0}$, and $Z_{1}$ and are determined based on stress-strain test data. The creep response constants are $Z_{i}, r$, and $A$, and are determined based on data from at least two creep tests at two different stress levels. Step-by-step theoretical evaluation of the material parameters was developed by Bodner and Partom (1975) for Rene 95, and by Stouffer (1981) for In 100. Also, Stouffer and Bodner (1981) studied the relationship between theory and experiment for the state variable constitutive equation. Therefore, to complete the study of the constitutive equations, a numerical evaluation of the material parameters was undertaken.

\section{Numerical Evaluation of Material Parameters}

A numerical study of a nonlinear time dependent material was undertaken, where the material variables were calculated using a direct curve fitting technique.

In general the Bodner material parameters are temperature dependent, but by performing the material characterization tests (stress-strain and creep) at the same temperature that the Bodner model will be applied, this temperature dependence is suppressed.

To determine the Bodner variables $\left(n, Z_{0}, Z_{1}, m, A, r, Z_{i}\right.$, and $\left.D_{0}\right)$, we consider measured and simulated plastic strain. For the evaluation of simulated $\dot{\epsilon}^{p}(t)$, the total strain rate is considered the sum of elastic and plastic strain rates

$\dot{\boldsymbol{\epsilon}}^{t}(t)=\dot{\boldsymbol{\epsilon}}^{e}(t)+\dot{\boldsymbol{\epsilon}}^{p}(t)$

with

$\dot{\boldsymbol{\epsilon}}^{e}(t)=\frac{\dot{\sigma}(t)}{E}$ 
One can rewrite Eq. 11 in the form

$\dot{\boldsymbol{\epsilon}}^{p}(t)=\dot{\epsilon}^{t}(t)-\frac{\dot{\sigma}(t)}{E}$

with $\dot{\sigma}(t)$ extracted from the data.

For the evaluation of theoretical $\dot{\boldsymbol{\epsilon}}^{p}$, one uses the flow law:

$\dot{\epsilon}^{p}(t)=\lambda(X) S$

where $S=$ deviatoric stress; and $\lambda=$ scalar function of the state of the material, $\lambda(X)=\lambda\left(n, Z_{0}, Z_{1}, m, A, r, Z_{i}, D_{0}, Z, \sigma\right)$. Using Eq. 14 and integrating Eq. 11 we get

$\epsilon^{t}(t)=\epsilon^{t}(0)+\int \dot{\epsilon}^{p}(t) d t+\frac{\sigma(t)}{E}$

$\epsilon^{\prime}(0)$ is taken from the data.

A sum square error function is defined

$Q=\sum_{\text {data }} w(t)\left[\epsilon^{\prime}(t)_{\operatorname{sim}}-\epsilon^{\prime}(t)_{\text {data }}\right]^{2}$

where $w(t)=$ positive weight function; and $\left[\epsilon^{t}(t)_{\text {sim }}-\epsilon^{t}(t)_{\text {data }}\right]=$ point time domain strain error. $Q$ was minimized in a computerized scheme by varying values of the material coefficients. In this analysis, a Runge-Kutta (fourth order) algorithm was employed for the numerical time integration of the Bodner equations in the following system:

$D_{2}^{p}=D_{0}^{2} \exp \left[\left(-\frac{Z^{2}}{3 J_{2}}\right)^{n}\left(\frac{n+1}{n}\right)\right]$

$\lambda=\left[D_{2}^{p} J_{2}\right]^{1 / 2}$

$\dot{\epsilon}^{p}=\lambda S$

$\dot{Z}=m\left(Z_{1}-Z\right) \sigma \dot{\epsilon}^{p}+\dot{Z}_{r}$

where $J_{2}$ is the second stress invariant.

Using a variant of Powell's (1964) search algorithm and the sum square error function, the specific material variables for the Bodner model were determined to best fit the tensile and creep data.

\section{EXPERIMENTAL DATA}

In the experimental part of this research, a group of tensile and creep tests were performed for Inconel 718 at $650^{\circ} \mathrm{C}\left(1,200^{\circ} \mathrm{F}\right)$. The Bodner model was employed to determine the material variables of Inconel 718 to best fit this data.

To help eliminate inconsistencies in data, the same specimen geometry was used for all tests. A drawing of the Button-Head specimen used in this study is presented in Fig. 1.

All experiments were performed in an electro-hydraulic testing machine equipped with a special high-temperature furnace. Special attention was given to the alignment of the specimen to minimize the eccentricity of the load 


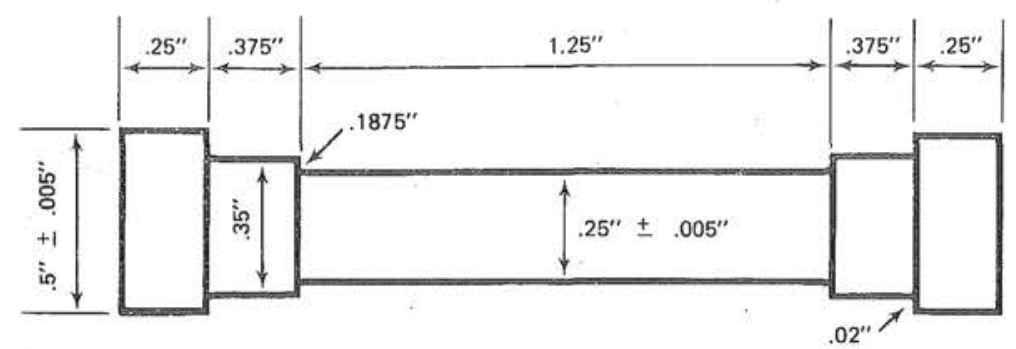

FIG. 1. Dimensions of Button-Head Test Specimen

and to obtain a uniform temperature profile in the test section. The machine was run under strain control. The data (stress, strain, time) was obtained by using the interferometric strain/displacement gage (ISDG) technique developed and conducted by W. N. Sharpe (Sharpe and Martin 1978).

\section{Results and Conclusions}

Uniaxial tensile tests were run at strain rates of $1.6 \times 10^{-3}, 0.67 \times 10^{-4}$, $1.0 \times 10^{-5}, 1.1 \times 10^{-6}$, and $3.3 \times 10^{-7} \mathrm{~s}^{-1}$. Creep tests were run at 758 and $862 \mathrm{MPa}$. The data for each test was smoothed and approximately 20 data points were used from each test in the fitting procedure. The modulus of elasticity was considered as a parameter, increasing the number of constants to be determined to nine. Different starting values were tried, producing slightly different values of constants though no appreciable difference in the agreement with individual curves. A unique set of constants was not determined.

The final parameters determined are listed in Table 1 where the values from Milly (1982) are also listed. Figs. 2 and 3 show the response curves from the model and the experimental data (only two of the five tensile tests are shown in Fig. 2). Agreement in Fig. 2 is reasonable except for the fastest tensile test, $2 b$. Agreement between model and data does not appear to be good in Fig. 3, but it is considerably better than other comparisons (Stouffer 1981; Milly 1982). The model modulus of elasticity of $172 \times 10^{3} \mathrm{MPa}$ is high for this material at this temperature. Other determinations place it at

TABLE 1. Bodner Parameters for Inconel 718 at $650^{\circ} \mathrm{C}\left(1,200^{\circ} \mathrm{F}\right)$

\begin{tabular}{c|c|c|c}
\hline \hline $\begin{array}{c}\text { Constant } \\
(1)\end{array}$ & $\begin{array}{c}\text { Units } \\
(2)\end{array}$ & $\begin{array}{c}\text { Milly and Allen (1982) } \\
(3)\end{array}$ & $\begin{array}{c}\text { This work } \\
(4)\end{array}$ \\
\hline$N$ & - & 1.167 & 0.7374 \\
$Z_{0}$ & $\mathrm{MPa}$ & 3,130 & 6.520 \\
$Z_{1}$ & $\mathrm{MPa}$ & 4,140 & 7,030 \\
$m$ & $\mathrm{MPa}$ & $2.43 \times 10^{-2}$ & $6.86 \times 10^{-1}$ \\
$A$ & $\mathrm{~s}^{-1}$ & $1.1 \times 10^{-4}$ & $6.82 \times 10^{-4}$ \\
$R$ & - & 2.857 & 4.734 \\
$Z_{i}$ & $\mathrm{MPa}$ & 2,760 & 3,690 \\
$D_{0}$ & $\mathrm{~s}^{-1}$ & $10^{4}$ & $1.03 \times 10^{4}$ \\
$E$ & $\mathrm{MPa}$ & $165 \times 10^{3}$ & $172 \times 10^{3}$ \\
\hline \hline
\end{tabular}



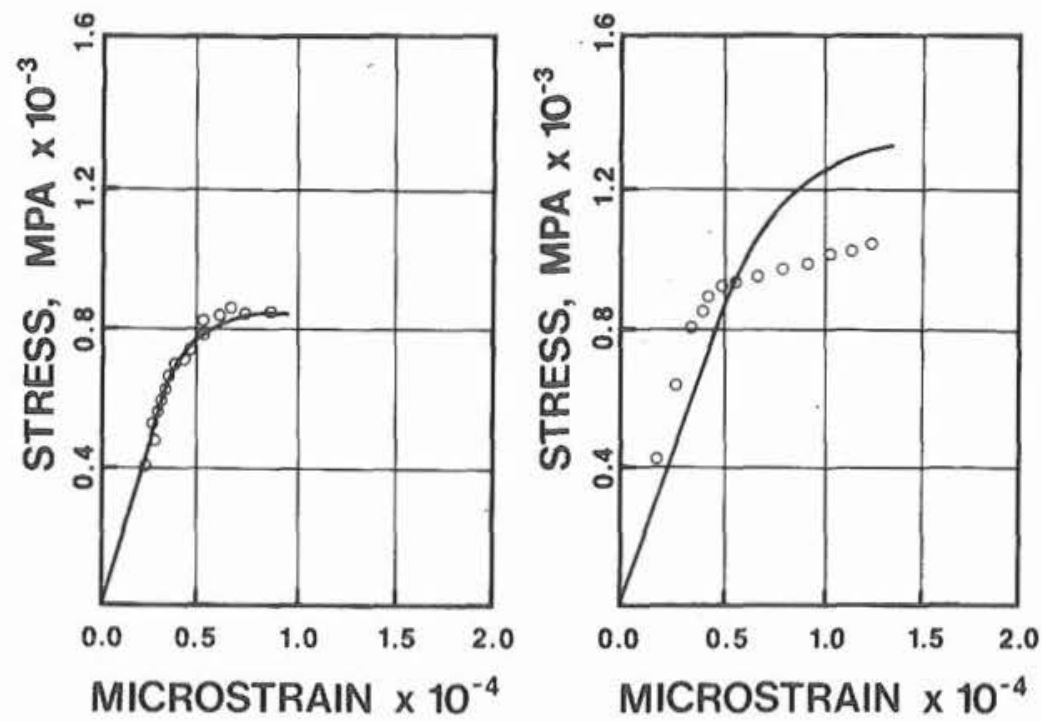

FIG. 2. Comparison of Stress-Strain with Predictions of Bodner Model; Cylindrical Specimens of Inconel 718 at $650^{\circ} \mathrm{C}$ : (a) $\dot{\epsilon}=3.3 \times 10^{-7} \mathrm{~s}^{-1} ;($ b) $\dot{\epsilon}=1.6 \times$ $10^{-3} \mathrm{~s}^{-1}$
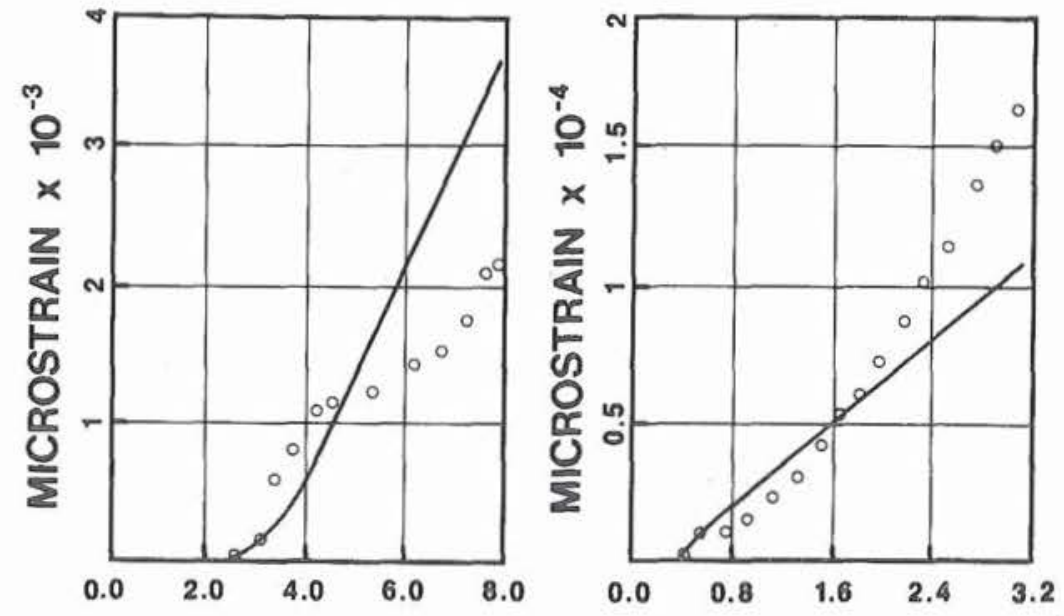

TIME SECONDS $\times 10^{-4}$ TIME SECONDS $\times 10^{-4}$

FIG. 3. Comparison of Creep Results with Predictions of Bodner Model; Cylindrical Specimens of Inconel 718 at $650^{\circ} \mathrm{C}$ : (a) Stress $=758 \mathrm{MPa}$; (b) Stress $=862$ MPa 
$155 \times 10^{3} \mathrm{MPa}$ (3). As can be observed from Fig. 2, the modulus for the experimental data is higher still at the faster rates. Nevertheless, the Bodner model describes the results fairly well given the wide range of strain rates.

This numerical scheme for identifying the Bodner parameters is straightforward and tends to give better agreement with the response curves than graphical methods. The Bodner model does reasonably well in describing stress-strain curves covering a wide range of strain-rates (almost $10^{4}$ in these experiments) and creep curves. Actually, it is a stiff requirement to ask a model to cover such a wide variety of material response. The parameters given were determined from the collective data over all of the tests, tensile and creep. When each test was used separately to determine a parameter set, excellent fitting was observed. This suggests there may have been some test to test variation in material properties.

The eight parameters of the Bodner model give it the requisite range, but a unique set of parameters was not obtained. In general, one can make the following conclusions:

1. A numerical evaluation of the material variables can be made by using Bodner constitutive theory through a numerical simulation of the tensile and creep response with reasonable curve fitting to the experimental response.

2. Bodner constitutive theory is very sensitive to the variability of the experimental data. Since the stress, $\sigma$, is the driving force in the constitutive model, a special attention should be given to the time data for a smooth $(\sigma-t)$ curve.

3. As in Bodner and Partom (1975), Bodner constitutive theory may need further work to decide on improvements which can be made to include effects that would lead to tertiary creep in the representations.

4. From the fact that the calculated values for some of the variables are different for the same value of fitting error (and they should not be) and from the fact that for some runs $Z_{0}$ and $Z_{1}$ have the same value (and they should not), it can be recognized that the material variables in Bodner's constitutive model are not well-defined. More specifically, they are not unique, which may address the fact that $650^{\circ} \mathrm{C}\left(1,200^{\circ} \mathrm{F}\right)$ is quite near a transition temperature for this material.

\section{Appendix I. References}

Bodner, S. R., and Partom, Y. (1975). "Constitutive equation for elastic-viscoplastic strain hardening materials." J. Appl. Mech., ASME, 42, 385-389.

Hinnerichs, T., Nicholas, T., and Pulayatlo, A. (1982). "A hybrid experimentalnumerical procedure for determining creep crack growth rates." Engrg. Fracture Mech., 16, 265-277.

Milly, T. M., and Allen, D. H. (1982). "A comparative study of nonlinear ratedependent mechanical constitutive theories for crystalline solids at elevated temperatures." Report AP1-E-5-82-5, Virginia Polytechnic Institute and State University, Blacksburg, Va., Mar.

Powell, M. H. D. (1964). "An effective method for finding the minimum of a function of several variables without calculating the derivatives." Computer J., 7, 144 162.

Sharpe, W. N., and Martin, D. R. (1978). "Optical measurement of in-plane strain/ displacement near tips at high temperature." Sixth Int'l Cong. on Experimental Stress Analysis, Munich.

Stouffer, D. C. (1981). "A constitutive representation for IN-100." Air Force Wright Aeronautical Laboratories, AFWAL-TR-81-4039, Wright-Patterson Air Force Base, Dayton, Ohio. 
Stouffer, D. C., and Bodner, S. R. (1981). "A relationship between theory and experiment for a state variable constitutive equation." AFWAL-TR-80-4194, Air Force Wright Aeronautical Laboratories, Wright-Patterson Air Force Base, Dayton, Ohio.

\section{APPENDIX II. NotATION}

The following symbols are used in this paper:

$A=$ coefficient controlling the rate of hardening recovery;

$D_{0}=$ constant representing the limiting value of the plastic strain rate in shear;

$E=$ Young's modulus of elasticity;

$J_{2}=$ second stress invariant;

$m=$ material constant controlling the rate of work hardening;

$n=$ constant related to the viscosity of the dislocation motion; it controls the strain rate sensitivity;

$Q=$ square error function;

$r=$ exponent controlling rate of hardening recovery;

$S=$ deviatoric stress;

$t=$ time;

$W=$ work;

$w=$ positive weight function;

$Z=$ plastic state variable measure of the overall resistance to plastic flow, hardness;

$\epsilon=$ current value of strain;

$\lambda=$ scalar function of the state of the material; and

$\sigma=$ current value of stress.

\section{Subscripts}

$0=$ initial value of $Z$;

$1=$ saturation value of $Z$; and

$i=$ state variable value of $Z$.

$$
\begin{aligned}
& \text { Superscripts } \\
& \begin{aligned}
e & =\text { elastic; } \\
p & =\text { plastic; } \\
t & =\text { total; } \\
& =\text { time derivative. }
\end{aligned}
\end{aligned}
$$

